\title{
Amoebic Cerebral Abscess: Still A Diagnostic Dilemma with Fatal Outcome
}

\author{
Akriti Jindal ${ }^{1}$, Ruchita Tyagi ${ }^{1 *}$, Avantika Garg ${ }^{1}$, Gursheen Kaur², Ashwani Kumar Chaudhary ${ }^{3}$, \\ Gagandeep Singh ${ }^{4}$, Harpreet Kaur ${ }^{1}$ and Neena Sood ${ }^{1}$ \\ ${ }^{1}$ Department of Pathology, Dayanand Medical College \& Hospital, Tagore Nagar, Ludhiana, India \\ ${ }^{2}$ Department of Cardiology, Institute of Medical Sciences, Banaras Hindu University, Varanasi, Uttar Pradesh, India \\ ${ }^{3}$ Department of Neurosurgery, Dayanand Medical College \& Hospital, Tagore Nagar, Ludhiana, India \\ ${ }^{4}$ Department of Neurology, Dayanand Medical College \& Hospital, Tagore Nagar, Ludhiana, India
}

\begin{abstract}
Amoebiasis is defined as infection with Entamoeba histolytica, which enters the body through feco - oral route after consumption of food or water contaminated by cysts. We present a rare case of amoebic brain abscess in a 45 years old patient who came with headache, fever and right sided weakness for 15 days. He was emperically being treated for tuberculosis. As Entamoeba was not suspected, no serological or microbiological investigations for the same were done. Histopathological examination of abscess sent after frontal lobectomy revealed trophozoites of Entamoeba histolytica. Demonstration of trophozoites of Entamoeba on histopathology sections was a surprising finding which clinched the diagnosis in this case. Amoebic brain abscess should be considered as differential diagnosis in all patients who present with signs \& symptoms of central nervous system involvement in endemic areas. Early diagnosis and aggressive management with metronidazole is imperative to limit the morbidity and prevent the mortality.
\end{abstract}

Keywords: Abscess, Amoebiasis, Cerebral, Entamoeba

\section{Introduction}

Entamoeba histolytica is a parasite commonly found in Central and South America, Africa, and the Indian subcontinent. [1] It usually causes colitis while extra intestinal infection results in abscesses in liver, lung and central nervous system (CNS). Brain abscess due to E. histolytica may turn fatal if not detected and treated timely. [2-6]

We present a rare case of amoebic brain abscess with review of literature. Cerebral amoebiasis was diagnosed on histopathological evaluation of necrotic tissue biopsied from frontal lobe. Fatal outcome of brain abscess due to Entamoeba histolytica is an unfortunate and unusual occurrence in today's era of advanced medical technology. Inspite of the availability of a plethora of diagnostic tools, histopathology and microbiology are still the cornerstone of diagnosis of this rare complication of amoebic infection.

\section{Case Report}

A 45 years old male, presented with high grade fever with chills and rigors for 2 months, bitemporal headache and weakness of right side body for 15 days. He was on emperical anti tubercular treatment for one month. He had altered sensorium and vomiting one day prior to admission. MRI revealed hyperintense lesions in bilateral frontal lobes, with acute infarct of genu of corpus callosum and left basal ganglia along with right cerebellar abscess. (Figure 1) The differential diagnoses included chronic meningitis due to tuberculosis, fungal abscess and infarction due to tubercular vasculitis. Sputum smear for Acid fast bacilli was negative. Right frontal lobectomy was performed and on opening, both the frontal lobes had undergone liquefactive necrosis. The necrotic material was sent for histopathological examination. Microscopy revealed numerous trophozoites of Entamoeba histolytica surrounded by clear space present in the brain parenchyma with areas of extensive necrosis. (Figures 2 a, 3) Surrounding areas showed dense infiltration by inflammatory infiltrate composed of neutrophils and foamy macrophages. (Figure 2 b) Erythrophagocytosis was also evident within the trophozoites which were highlighted by Phospho Tungstic Acid Hematoxylin (PTAH) (Figure 3 inset) and Periodic Acid Schiff (PAS) stains. (Figure 2 b) On the basis of histology, diagnosis of amoebic brain abscess was made. $\mathrm{KOH}$ preparation of biopsy tissue showed no growth. No culture reports were available.

Even though the diagnosis was informed to the clinician at the earliest, the patient succumbed to the fulminant infection before the treatment could be started.

\section{Discussion}

This was a case of cerebral amoebiasis, a fatal complication of Amoebic infection, diagnosed on histopathology of frontal lobe biopsy. Amoebiasis is defined as infection with 


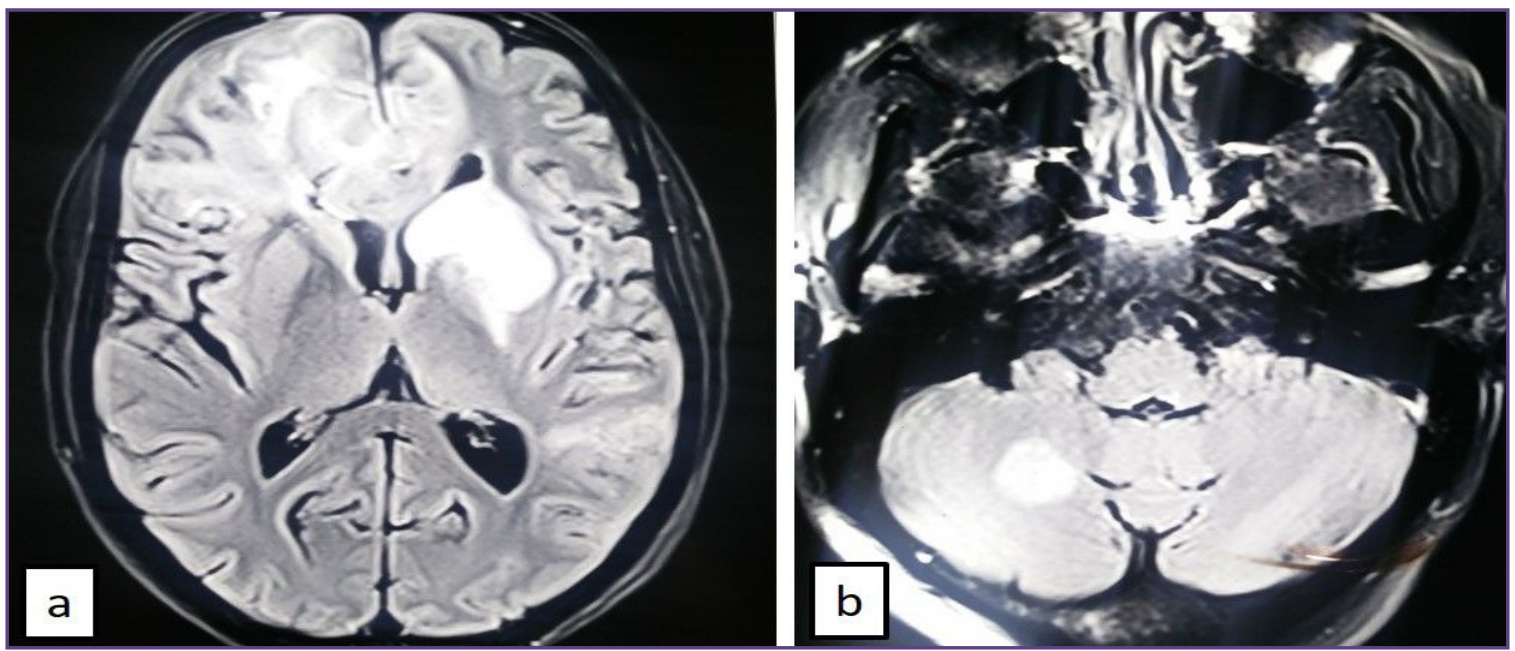

Fig. 1: MRI showing a) hyperintense lesions in bilateral frontal lobes, with acute infarct of genu of corpus callosum; b) right cerebellar abscess.

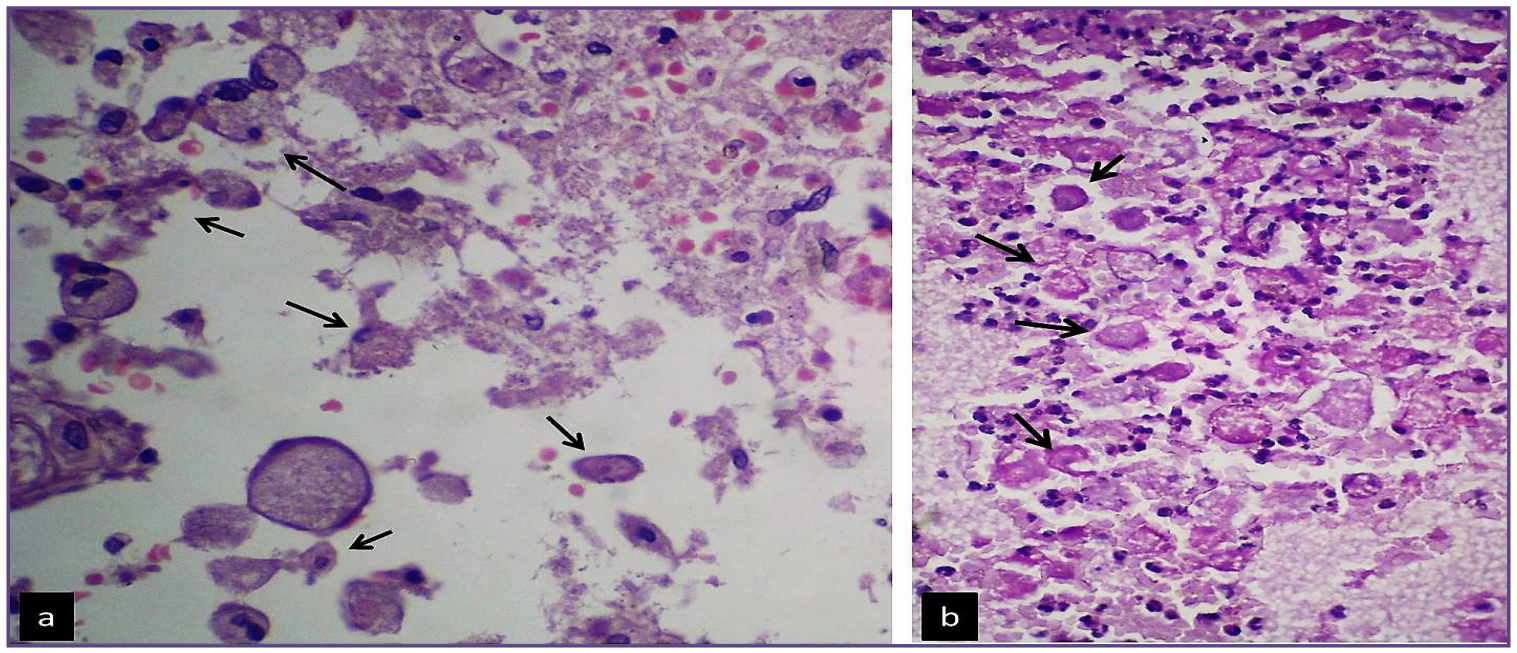

Fig. 2: a) Trophozoites (black arrows) displaying erythrophagocytosis in areas of necrosis (H\&E,400x); b) PAS stain highlighting trophozoites (black arrows) in necrotic areas containing neutrophils.(400x).

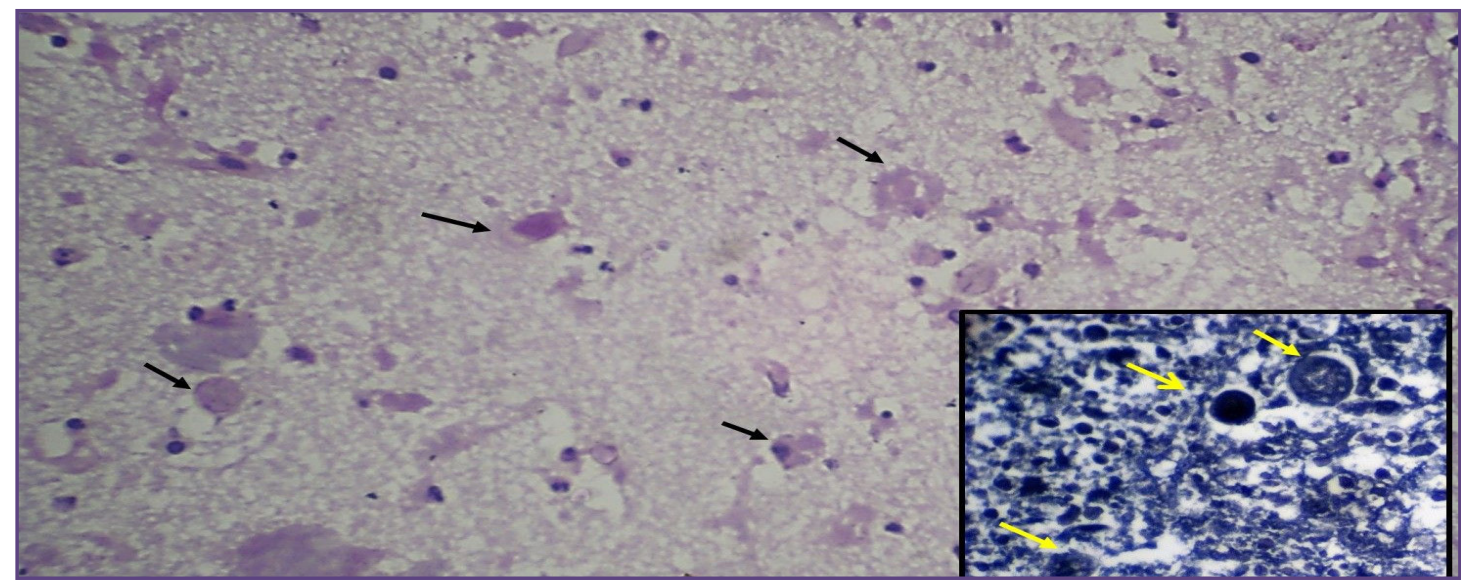

Fig. 3 : Photomicrograph showing trophozoites of Entamoeba (black arrows) in the cerebral parenchyma.(H\&E, 100x). Inset shows Entamoeba trophozoites (yellow arrows) on PTAH stain. 
E. histolytica, regardless of symptomatology. [7] Although both genders and all ages are affected; males are more commonly affected than females. [8] The disease spreads through feco - oral route by consumption of food or water contaminated by cysts of Entamoeba which survive the acidic environment of the stomach [1 - 4]. Trophozoites can invade the intestinal mucosa, causing amoebic colitis and invade the mucosa to enter the bloodstream and infect the extra intestinal sites. Cerebral involvement in the form of a brain abscess occurs in less than $0.1 \%$ cases having liver abscess.[9] The other amoebae that can infect the brain are Acanthamoeba, Balamuthia, Sappinia, and Naegleria, which can cause granulomatous amoebic encephalitis mostly in immunocompromised people.[10] Amoebic brain abscess is a rare complication of Entamoeba histolytica infection, which may have a fatal outcome if not detected early and aggressively treated. Common presentations include headache, sensory disturbances and features of meningitis like vomiting and altered mental status. [4, 6,10 - 12,14] Cerebral abscess may be single, multiple or confluent. [10 - 13] In this case, multiple lesions were present. Microscopically, there may be granulomatous response associated with abscess formation containing central necrotic core and peripheral encapsulation. [14]

Serological investigation includes ELISA test with values ranging from 1:1000 to 1:50000 (dilution) which suggests extra - intestinal amoebiasis. Serum antibodies reflect amoebic invasion but do not correlate with protective immunity. [10] Radiology can demonstrate presence of abscesses, infarcts and other lesions but etiology can be confirmed only on demonstration of the parasite on histopathological or microbiological examination. Diagnosis is difficult due to resemblance of clinical symptoms with brain tumor and meningitis while radiological features may mimic tubercular or fungal infection. In this case also, the patient was suspected to have tuberculosis or superimposed fungal infection and empirically, put on anti - tubercular treatment. Polymerase Chain Reaction of aspirated material from brain abscess has also been used to diagnose cerebral amoebiasis. [3, 6] As Entamoeba was not suspected, no serological or microbiological investigations for the same were done in this case. Demonstration of trophozoites of Entamoeba on histopathology sections was a surprising finding which clinched the diagnosis.

Metronidazole is the drug of choice for treating amoebiasis. $[3,14,15]$ The duration of treatment may range from two to eight weeks, whenever the abscess resolves. Surgical intervention may be in the form of pus drainage or total excision of the abscess wall and is indicated in cases of a large, multiloculated abscess, no response to antibiotics, mass effect, poor Glasgow Coma Scale score, and development of new abscesses.[11, 13, 14, 15] But our patient succumbed to the fuminant infection before the drugs could be administered.

\section{Conclusion}

Amoebic brain abscess should be considered as differential diagnosis in all patients who present with signs \& symptoms of central nervous system involvement in endemic areas. Cerebral amoebiasis is characterized by rapid progression and early death if untreated. It poses a therapeutic challenge as evidenced by the paucity of papers reporting complete recovery after treatment. Early diagnosis and aggressive management is imperative to limit the morbidity and prevent the mortality.

\section{Reference}

1. Singh U, Petri WA Jr. Amoebas. In: Gillespie SH, Pearson RD, editors. Principles and Practice of Clinical Parasitology. Chichester: John Wiley \& Sons, Ltd.; 2001. p. 197-218.

2. Chatterjee KD, editor. Subphylum sarcomastigophora: Superclass sarcodina, class rhizopodea: Order amoebida. In: Parasitology (Protozoology and Helminthology) in Relation to Clinical Medicine. 12th ed. Kolkata, India: SreeSaraswaty Press Ltd; 2001. p. 14-36.

3. Petri WA, Haque R. Entamoeba histolytica brain abscess. Handb Clin Neurol. 2013;114:147-52. doi: 10.1016/B9780-444-53490-3.00009-1.

4. Viriyavejakul P, Riganti M. Undiagnosed amebic brain abscess. Southeast Asian J Trop Med Public Health 2009;40:1183-7.

5. Maldonado-Barrera CA, Campos-Esparza Mdel R, MuñozFernández L, Victoria-Hernández JA, Campos-Rodríguez R, Talamás-Rohana $\mathrm{P}$, et al. Clinical case of cerebral amebiasis caused by E. histolytica. Parasitol Res 2012;110:1291-6. doi: 10.1007/s00436-011-2617-8. Epub 2011 Aug 26.

6. Ohnishi K, Murata M, Kojima H, Takemura N, Tsuchida T, Tachibana H. Brain abscess due to infection with Entamoeba histolytica. Am J Trop Med Hyg 1994;51:180-2.

7. Ximénez C, Morán P, Rojas L, Valadez A, Gómez A. Reassessment of the epidemiology of amoebiasis: State of the art. Infect Genet Evol 2009;9:1023-32.

8. Gupta KB, Manchanda M, Chaudhary U, Verma M. Superior vena cava syndrome caused by pulmonary amoebic abscess. Indian J Chest Dis Allied Sci 2006;48:275-7.

9. Stanley SL Jr. Amoebiasis. Lancet 2003;361:1025-34.

10. Kothiwala AK, S Rai HI, Garg K, Singh M, Singh P, Sharma BS, Sood R. Giant anterior interhemispheric fissure amoebic abscess: A rare case. Neurol India 2018;66:548-51.

11. Sayhan Emil S, Altinel D, Bayol U, Ozcolpan OO, Tan A, Ganiusmen O. Indian J Pediatr 2008;75:1078-80. https://doi. org/10.1007/s12098-008-0182-7 
12. Tamer GS, Öncel S, Gökbulut S, Arisoy ES. A rare case of multilocus brain abscess due to Entamoeba histolytica infection in a child. Saudi Med J 2015;36:356-8.

13. Sundaram C, Prasad BC, Bhaskar G, Lakshmi V, Murthy JM. Brain abscess due to Entamoeba histolytica. J Assoc Physicians India 2004;52:251-2.
14. Sarica FB, Tufan K, Cekinmez M, Erdogan B, Altinors MN. A Rare But Fatal Case of Granulomatous Amebic Encephalitis with Brain Abscess: The First Case Reported from Turkey Turkish Neurosurgery 2009;19:256-9.

15. Morishita A, Yamamoto H, Aihara H. A case of amebic brain abscess. [Article in Japanese] No Shinkei Geka. 2007;35:919-25.

*Corresponding author:

Dr. Ruchita Tyagi, Assistant Professor, Department of Pathology, Dayanand Medical College \& Hospital, Tagore Nagar, Ludhiana, Punjab, India. Phone: +91 9876589911

Email: ruchitatyagi@gmail.com

Financial or other Competing Interests: None. 